\title{
Pediatric LGI1 and CASPR2 autoimmunity associated with COVID 19: Morvan syndrome
}

\author{
Mehmet Akif Kilic ${ }^{1} \cdot$ Zeynep Nagihan Yoruk Yildirim ${ }^{2} \cdot$ Adil Oner $^{2} \cdot$ Elif Yesil $^{2} \cdot$ Bagdagül Aksu $^{2}$. \\ Edibe Pembegul Yildiz ${ }^{1,3} \cdot$ Alev Yilmaz ${ }^{2}$. Meliha Mine Caliskan ${ }^{1,3}$
}

Received: 22 April 2021 / Revised: 11 May 2021 / Accepted: 12 May 2021 / Published online: 18 May 2021

○) Springer-Verlag GmbH Germany, part of Springer Nature 2021

\section{Dear Sirs,}

Although severe acute respiratory syndrome coronavirus 2 (SARS-CoV-2) most commonly affects the respiratory system, autoimmune neurological presentations have also been reported [1,2]. More recently Burr et al. have reported a pediatric patient with SARS-CoV-2-associated antiNMDAR encephalitis [1]. Although anti-NMDAR encephalitis is one of the most common types of autoimmune encephalitis (AIE) leucine-rich glioma-inactivated protein 1 (LGI1) and contactin-associated protein 2 (CASPR2)-associated autoimmunity is rare in children. LGI1 and CASPR2 are the main antigens within the voltage-gated potassium channel-complex (VGKC). LGI1 and CASPR2 autoimmunity is associated with limbic encephalitis, isolated epilepsy, Morvan syndrome and neuromyotonia [3]. Morvan syndrome is characterized by neuromyotonia, dysautonomia, insomnia and neuropsychiatric symptoms [3, 4]. Here we present the pediatric patient with SARS-CoV-2-associated Morvan syndrome initially present with Guillain-Barre syndrome.

A previously healthy 3-year-old girl has admitted to an outside hospital for a history of sleep dysregulation and irritability followed by lower extremities weakness with difficulty in walking for 2 months ago. Her first examination had revealed tachycardia, arterial hypertension, ataxia and abolished deep tendon reflexes of the lower extremities. She had family history of coronavirus disease 2019 (COVID-19) 4 weeks before her symptoms developed. She

Edibe Pembegul Yildiz

edibepembegul@hotmail.com

1 Department of Pediatric Neurology, Istanbul Medical Faculty, Fatih/İstanbul, Turkey

2 Department of Pediatric Nephrology, Istanbul Medical Faculty, Fatih/İstanbul, Turkey

3 Istanbul University Institute of Child Health, Istanbul, Turkey was tested for screening of COVID-19. SARS-CoV-2 RealTime Reverse Transcriptase (rRT)-PCR was detected negative on nasopharyngeal swab. SARS-CoV-2 IgG antibodies (ab) from serum were positive. Initial blood chemistry has only revealed mild hyponatremia. CSF analysis demonstrated albuminocytologic dissociation. GBS has been presumed and intravenous immunoglobulin (IVIG) $2 \mathrm{~g} / \mathrm{kg}$ was administered. Patient received antihypertensive treatment with three medications (metoprolol, enalapril, and prazosin), which showed limited benefit for control of elevated blood pressure. Four weeks following IVIG administration she was still agitated and had no recovery of weakness. Furthermore, she developed hyperhidrosis and pruritus and severe hypertension persisted despite the triple antihypertensive medication.

Patient refeered to our hospital one month after IVIG administration. She had ataxia, sleep dysregulation, nocturnal restlessness, episodes of crying, irritability, hyperhidrosis, tachycardia, and severe arterial hypertension. SARSCoV-2 IgG and IgM-ab from serum were positive. Blood chemistry showed; mild hyponatremia with $133 \mathrm{mmol} / \mathrm{L}$ and increased serum levels of free thyroxine (fT4). Repeated CSF analysis demonstrated albuminocytologic dissociation (Table1). Extended microbiology and oncology screening on blood, bone marrow, and CSF was unremarkable. MRI of the brain with and without contrast, abdominal ultrasound, renal doppler ultrasound, abdominal CT angiography, chest CT, and chest X-Ray were normal. Spine MRI revealed diffuse thickening and enhancement of cauda equina nerve roots. Electroencephalography showed diffuse slowing of the background activity. EMG could not performed. Antineural antibody screening revealed LGI1 (serum titer 1:80) and CASPR2-ab (serum titer 1:160), done by a commercial cellbased assay (Euroimmun, Lubeck, Germany) with a modified protocol. CSF analysis for CASPR2 and LGI1-ab were negative. Ten days after admission to our hospital repeated serum LGI1 and CASPR2-ab remained positive (1/80 and 
Table 1 Summary of laboratory tests at 3 time points

\begin{tabular}{|c|c|c|c|c|}
\hline & $\begin{array}{l}\text { Time 1: } 1-10 \mathrm{D} \text { prior } \\
\text { to IVIG }\end{array}$ & $\begin{array}{l}\text { Time 2: 30th D follow- } \\
\text { ing IVIG }\end{array}$ & $\begin{array}{l}\text { Time } 3 * \text { : 40th D follow- } \\
\text { ing IVIG }\end{array}$ & Reference Range \\
\hline \multicolumn{5}{|l|}{ Spinal fluid analysis } \\
\hline White blood cell count & 2 & & 0 & $0-5$ \\
\hline Glucose (mg/dL) & & & 62 & $40-70$ \\
\hline Protein $(\mathrm{mg} / \mathrm{dL})$ & 118 & & 61.8 & $15-45$ \\
\hline Albumin (mg/dL) & 73.6 & & 55.1 & $5-25$ \\
\hline $\operatorname{IgG}(\mathrm{mg} / \mathrm{L})$ & 274 & & 75 & $3-30$ \\
\hline IgG index & 1.7 & & 0.52 & $<0.7$ \\
\hline Oligoclonal bands (OCBs) & & & No OCBs present & \\
\hline HSV 1, HSV 2 PCR & & & Negative & \\
\hline HHV 6,7,8 PCR & & & Negative & \\
\hline Enterovirus PCR & & & Negative & \\
\hline EBV, CMV, VZV PCR & & & Negative & \\
\hline \multicolumn{5}{|l|}{ Blood tests } \\
\hline Anti-AMPA1/AMPA2 & & Negative & Negative & \\
\hline Anti-NMDA & & Negative & Negative & \\
\hline Anti GABA- B & & Negative & Negative & \\
\hline Anti-CASPR2 & & Positive (1/160) & Positive (1/320) & \\
\hline Anti-LGI1 & & Positive (1/80) & Positive (1/80) & \\
\hline $\begin{array}{l}\text { Anti-Yo, Anti-Hu, Anti-Ri, Anti-Ma2 Anti- } \\
\text { CV2 }\end{array}$ & & Negative & Negative & \\
\hline SARS-CoV-2 (rRT)-PCR & Negative & Negative & & \\
\hline SARS-CoV-2 IgG & Positive & Positive & Positive & \\
\hline SARS-Cov-2 IgM & & Positive & Negative & \\
\hline fT4 (pmol/L) & & 28.4 & & $11-22.5$ \\
\hline TSH (mIU/L) & & 2.2 & & $0.54-4.53$ \\
\hline Anti-TPO Ab (IU/ml) & & 18.2 & & $0-34$ \\
\hline Comploment component $3(\mathrm{mg} / \mathrm{dL})$ & & 151.6 & & $70-180$ \\
\hline Comploment component 4 (mg/dL) & & 25.5 & & $10-40$ \\
\hline ENA** & & Negative & & \\
\hline $\mathrm{C}$ reactive protein $(\mathrm{mg} / \mathrm{L})$ & & 0.3 & 0.6 & $0-5$ \\
\hline
\end{tabular}

*One day prior to IV methylprednisolone treatment

**ENA Anti-Ro Ab, Anti-La Ab, Anti-jo-1 Ab, Anti-ScL70 Ab, Anti Sm Ab, Anti-RNP Ab

Abnormal test results are written in bold

$1 / 320$, respectively). We diagnosed combination of GBS and Morvan syndrome based on the clinical phenotype and laboratory findings. IV methylprednisolone (30 mg/ $\mathrm{kg} /$ day) was administered for 5 days and followed by oral prednisolone treatment. Over the following week, sleep dysregulation, nocturnal restlessness, episodes of crying, hyperhidrosis, pruritus and ataxia gradually resolved. Four weeks after IV methylprednisolone administration requirement of antihypertensive medication was reduced and tachycardia was improved. Moreover serum LGI1 and CASPR2-ab, which was repeated for the third time, were detected negative 3 weeks after IV methylprednisolone administration.

Morvan syndrome is a rare autoimmune disorder predominantly affects elderly men with the male to female ratio
19:1 in adult patients [5]. Unique feature of our patient is the female gender. To our knowledge 14 paediartic cases with Morvan syndrome and available antibody results were published and 5 cases were female [3, 4, 6-9]. According to the cases reported so far, the male predominance of Morvan syndrome in children is not as pronounced as in adults and this might possibly represent an age-dependent feature of the syndrome [3].

Serum studies of eight patients with Morvan syndrome have revealed increased serum levels of free thyroxine $(5 / 8)$, cortisol $(2 / 8)$, renin $(2 / 8)$, prolactin $(1 / 8)$, adrenalin/noradrenalin (3/8), isolated low triiodothyronine (1/8), and hyponatremia (1/8) [4]. Although hyponatremia has been infrequently reported in paediatric cases, was mildly 
present in our patient. Lee et. al have reported the different expressions of VGKC in hormone secreting neuronal nuclei within the hypothalamus in rats [10]. They have suggested this molecular diversity of VGKC may provide regulatory mechanisms in controlling endocrine and autonomic systems [10]. This report might explain the abnormalities of different hormonal axes in patients with Morvan syndrome $[4,10]$. In our case, serum levels of sodium and fT4 were detected in normal range, $138 \mathrm{mmol} / \mathrm{L}$ and $19.3 \mathrm{pmol} / \mathrm{L}$, respectively, 2 weeks after IV methylprednisolone administration.

In pediatric cases, CASPR2-ab-associated GBS has been reported previously [11]. The initial presentation of our patient has also suggested GBS. However, she had marked clinical deterioration despite IVIG administration and later developed clinical and laboratory features compatible with Morvan syndrome. In 2016, Lotan et al. have reported a 61-year-old man who had the combination of GBS-like and classic Morvan syndrome symptoms associated with the sole presence of LGI1-ab [12]. Our patient's clinical presentation was similar to their case. To our knowledge, this is the first pediatric case of confirmed Morvan syndrome presenting initially with GBS and this report broadens the clinical spectrum of LGI1 and CASPR2 autoimmunity.

Paraneoplastic association has been made with thymoma in adult patients with Morvan syndrome. Consistent with previously reported pediatric cases our patient had no thymoma which we ruled out with radiologic investigations. Moreover there also may be possible infectious triggers of the disease [13]. Several pediatric case reports have revealed the association between SARS-CoV-2 infection and the development of autoimmunity $[1,2,14]$. In our case, SARSCoV-2 IgG and IgM-ab were positive and suggesting that SARS-Cov-2 infection may triggered the CASPR2 and LGI1 autoimmunity.

In conclusion, we defined the relationship between combination of GBS and Morvan syndrome, which is the first report in children, and SARS-Cov-2 infection. Previously reported autoimmune neurologic manifestations in children with SARS-Cov-2 strengthens our theory.

\section{Declarations}

Conflicts of interest The authors declare that they have no conflict of interest.
Conflicts of interest This study has been carried out in accordence with the Declaration of Helsinki.

\section{References}

1. Burr T, Barton C, Doll E, Lakhotia A, Sweeney M (2021) N-Methyl-d-Aspartate receptor encephalitis associated with COVID-19 infection in a toddler. Pediatr Neurol 114:75-76

2. Gaughan M, Connoly S, O'Riordan S, Tubridy N, McGuigan C, Kinsella JA (2021) Pediatric parainfectious encephalitis associated with COVID-19. Neurology 96:541-544

3. Nosadini M, Toldo I, Tascini B, Bien CG, Parmeggiani L et al (2019) LGI1 and CASPR2 autoimmunity in children: systematic literature review and report of a young girl with Morvan syndrome. J Neuroimmunol 335:577008

4. Syrbe S, Stettner GM, Bally J, Borggrafae I, Bien CI, Ferfoglia RI et al (2020) CASPR2 autoimmunity in children expanding to mild encephalopathy with hypertension. Neurology 94:2290-2301

5. Masood W, Sitammagari KK (2018) Morvan syndrome (Morvan fibrillary chorea, MFC). StatPearls Publishing

6. Nikolaus M, Jackowski-Dohrmann S, Prüss H, Schuelke M, Knierim E (2018) Morvan syndrome associated with CASPR2 and LGI1 antibodies in a child. Neurology 90:183-185

7. López-Chiriboga AS, Klein C, Zekeridou A, McKeon A, Dubey D, Flanagan EP, Lennon VA, Tillema JM, Wirrell EC, Patterson MC, Gadoth A, Aaen JG, Brenton JN, Bui JD, Moen A, Otten C, Piquet A, Pittock SJ (2018) LGI1 and CASPR2 neurological autoimmunity in children. Ann Neurol 84:473-480

8. Rosenblatt T, Ort K, Shaw R, Levy RJ, Chen C, Niemi A, Hoang K (2020) A previously healthy adolescent with acute psychosis and severe hyperhidrosis. Pediatrics 145:1-7

9. Surana S, Kumar R, Pitt M, Hafner P, Mclellan A, Davidson J et al (2019) Acquired neuromyotonia in children with CASPR2 and LGI1 antibodies. Dev Med Child Neurol 61:1344-1347

10. Lee SK, Lee S, Shin SY, Ryu PD, Lee SY (2012) Single cell analysis of voltage-gated po- tassium channels that determines neuronal types of rat hypothalamic paraventricular nucleus neurons. Neuroscience 205:49-62

11. Rosch RE, Bamford A, Hacohen Y, Wraige E, Vincent A, Mewasingh L, Lim M (2014) Guillain-Barre syndrome associated with CASPR2 antibodies: two paediatric cases. J Peripher Nerv Syst 19:246-249

12. Lotan I, Djaldetti R, Hellman MA, Benninger F (2016) Atypical case of Morvan's syndrome. J Clin Neurosci 25:132-134

13. Maddison P, Lawn N, Mills KR, Vincent A, Donaghy M (1998) Acquired neuromyotonia in a patient with spinal epidural abscess. Muscle Nerve 21:672-674

14. de Miranda Henriques-Souza AM, de Melo ACMG, de Aguiar Coelho Silva Madeiro B, Freitas FL, Rocha-Filho SA, Gonçalves FG, (2020) Acute disseminated encephalomyelitis in a COVID-19 pediatric patient. Neuroradiology 63:141-145 